\title{
Inter-relações da produtividade de cana soca com a resistência à penetração, umidade e matéria orgânica do solo
}

\author{
Flávio Carlos Dalchiavon ${ }^{1}$, Morel de Passos e Carvalho², Rafael Montanari ${ }^{2}$, \\ Marcelo Andreotti ${ }^{2}$ Edjair Augusto Dal Bem ${ }^{3}$
}

\section{RESUMO}

A cultura da cana-de-açúcar desempenha, sobretudo no aspecto relacionado às fontes energéticas alternativas, importantíssimo papel. No ano de 2009, no município de Suzanápolis (SP), cujas coordenadas são 20²8' 10' 'S e 50 49' $20^{\prime}$ ' W, no cerrado brasileiro foi analisada a produtividade da cana soca ( $2^{\circ}$ ciclo) em razão da resistência à penetração, umidade gravimétrica e matéria orgânica de um Argissolo Vermelho eutrófico. O objetivo foi selecionar, entre os atributos pesquisados, aquele com a melhor correlação, a linear e a espacial, para explicar a variabilidade da produtividade da cana-de-açúcar. Foi instalada a malha geoestatística para a coleta de dados do solo e da planta, com 120 pontos amostrais, numa área de 14,53 ha. A matéria orgânica correlaciona-se linear e negativamente com a resistência à penetração, indicando que manejos do solo que visem seu aumento no perfil melhoram suas condições físicas e, por conseguinte, o desenvolvimento e a produtividade da cana-de-açúcar. A umidade gravimétrica (UG) e o teor de matéria orgânica do solo (MO) correlacionam-se direta, linear (UG2 e MO1) e espacialmente (UG1 e MO1) com a produtividade da cana-de-açúcar, demonstrando serem os melhores atributos, dentre os pesquisados, para estimar e aumentar a produtividade agrícola da cana-de-açúcar.

Palavras-chave: geoestatística, manejo e conservação do solo, Saccharum spp., variabilidade espacial.

\section{ABSTRACT}

\section{Relationship of sugarcane ratoon yeld with resistance penetration, humidity and soil organic matter}

The sugarcane crop plays an important role on Brazilian economy,, especially in the aspect related to alternative energy sources. Yield of ratoon cane (2nd cycle) was evaluated in relation to resistance to penetration, gravimetric moisture and organic matter in a Typic Tropustalf, in the municipality of Suzanápolis (SP), 20²8'10' S and 5049'20' W, in the Brazilian cerrado, in 2009. The main purpose was to select, among the attributes surveyed, the one with the highest linear and spatial correlations that explains the variability of sugar cane yield. A geostatistical grid was installed in order to collect data from the soil as well from the plant, with 120 sampling points in an area of 14.53 ha. Organic matter correlated linearly and negatively with penetration resistance, indicating that the soil management practices that aim its increase in the soil profile can improve soil physical conditions, and consequently, the development and yield of sugarcane. Both gravimetric moisture (UG) and content of soil organic matter (OM) correlated directly, linearly (UG2 and MO1) and spatially (UG1 and MO1) with sugarcane yield, proving to be the best attributes, among the evaluated ones, to estimate and increase the sugarcane yield.

Key words: geostatistical, soil management and conservation, Saccharum spp., spatial variability.

Recebido para publicação em 31/07/2012 e aprovado em 02/08/2013.

'Engenheiro-Agrônomo, Doutor. Instituto Federal de Educação, Ciência e Tecnologia de Mato Grosso - Campus Campo Novo do Parecis, MT 235 km 12; s/n, Caixa Postal n. 100, Bairro Zona Rural, CEP: 78360-000, Campo Novo do Parecis/MT. flavio.dalchiavon@cnp.ifmt.edu.br (autor para correspondência).

${ }^{2}$ Engenheiros-Agrônomos, Doutores. Departamento de Fitossanidade, Engenharia Rural e Solos, Faculdade de Engenharia de Ilha Solteira - UNESP, Caixa Postal 31, 15385-000, Ilha Solteira, São Paulo, Brasil. morel@agr.feis.unesp.br; montanari@agr.feis.unesp.br; dreotti@agr.feis.unesp.br

${ }^{3}$ Engenheiro Florestal. Doutor. Faculdade de Engenharia de Ilha Solteira - UNESP, Passeio Monção, 213, 15385-000, Ilha Solteira, São Paulo, Brasil. edjairflorestal@ hotmail.com 


\section{INTRODUÇÃO}

A cana-de-açúcar (Sacchharum spp.) é uma Poaceae semiperene. A sua importância socioeconômica para o Brasil é inquestionável, pois é a principal matéria-prima para a produção do etanol utilizado pelos veículos automotores, além da sua destinação para a produção do açúcar, um dos principais produtos de exportação nacional. De acordo com dados da Conab (2013), na safra 2012/13 o setor sucroalcooleiro nacional processou 588,9 milhões de toneladas de colmos, tendo o Estado de São Paulo como o maior produtor, participando com $56 \%$ do total (330,7 milhões de toneladas), numa área de 4,4 milhões de hectares, com a produtividade média em torno $74,8 \mathrm{t} \mathrm{ha}^{-1}$.

Muitas vezes, o preparo do solo é realizado sem levar em conta sua umidade, a qual é um fator controlador da compactação (Beutler \& Centurion, 2003). As principais alterações são evidenciadas pela diminuição da taxa de infiltração de água no solo e pelo aumento da resistência à penetração de raízes. A degradação dos atributos físicos decorrente do manejo inadequado condiciona decréscimo de produção da cana-de-açúcar (Utset \& Cid, 2001). Por outro lado, manejo visando à manutenção e/ou adição de matéria orgânica no solo é muito importante, uma vez que os benefícios são, principalmente, o aumento na resistência à erosão e na capacidade de armazenamento de água, devido a sua atuação sobre a estrutura do solo, por meio do aumento da estabilidade dos agregados, além da melhoria na fertilidade do solo.

A análise da variabilidade do solo, por meio da geoestatística, pode indicar alternativas de manejo para reduzir os seus efeitos sobre a produção das culturas (Trangmar et al., 1985; Montanari et al., 2012), estabelecendo zonas específicas de manejo visando à conservação do solo (p.e. aplicação de insumos com taxas variadas, descompactação das zonas compactadas), condições necessárias à agricultura de precisão (Montanari et al., 2005). Nos últimos anos, diversos trabalhos têm sido desenvolvidos com o propósito de se conhecer a inter-relação espacial entre os atributos do solo e a produtividade das culturas, merecendo destaque o de Souza et al. (2004a,b), Santos et al. (2006), Martins et al. (2009), Lima et al. (2010) e Dalchiavon et al. (2011), com as culturas do milho, feijoeiro, soja e eucalipto, respectivamente, tendo alcances geoestatísticos variando entre 13,9 e 169,0 m, representando a distância na qual os atributos apresentam valores iguais entre si.

Dessa forma, considerando o atual e substancial avanço da cultura canavieira no Brasil, o objetivo deste traba- lho foi estabelecer as inter-relações, lineares e espaciais, da produtividade de cana-de-açúcar com a resistência à penetração, umidade e matéria orgânica de um Argissolo Vermelho eutrófico do noroeste paulista.

\section{MATERIAL E MÉTODOS}

Este trabalho foi desenvolvido no noroeste do Estado de São Paulo, no município de Suzanápolis-SP, Fazenda Caiçara, Usina Vale do Paraná S/A Álcool e Açúcar, cujas coordenadas são $20^{\circ} 28^{\prime} 10^{\prime \prime}$ S e 5049'2 0' W. A precipitação média anual é de $1.300 \mathrm{~mm}$, enquanto a temperatura média do ar é de $23,7^{\circ} \mathrm{C}$. O tipo climático é Aw, segundo classificação de Köeppen, caracterizado como tropical úmido com estação chuvosa no verão e seca no inverno. O solo no qual a malha experimental foi instalada foi classificado como Argissolo Vermelho eutrófico típico textura arenosa/média, a moderado (Alves et al., 20094).

Para a implantação da cana-de-açúcar (variedade SP791011) em área de pastagem (braquiária) há 10 anos, foi necessário dessecá-la com herbicida glifosato na dose de $2,5 \mathrm{~kg}$ ha $^{-1}$ p.c. Para o preparo do solo foi realizada uma gradagem pesada e duas intermediárias (maio/2006), momento esse em que foram incorporados 2,0 $\mathrm{t} \mathrm{ha}^{-1}$ de calcário dolomítico (PRNT 85\%), aplicado a lanço, com incorporação, 0,35 m de profundidade, por arado de aivecas, finalizando o preparo com uma grade niveladora. Na sulcação adubou-se a área com $500 \mathrm{~kg} \mathrm{ha}^{-1}$ da fórmula 06-30-24 (NPK), e na cobertura dos toletes foi aplicado no sulco de plantio $250 \mathrm{~g} \mathrm{ha}^{-1}$ p.c. de fipronil, visando ao controle de pragas de solo.

O plantio foi realizado em 20 de junho de 2006, com espaçamento de 1,50 m. Após o primeiro corte da cultura, setembro de 2007 (colheita mecanizada), foram aplicados $1.000 \mathrm{~kg} \mathrm{ha}^{-1}$ de gesso. Na adubação da soqueira foram aplicados $500 \mathrm{~kg} \mathrm{ha}^{-1}$ da fórmula 18-00-27 (NPK). Para a coleta de dados, a cultura encontrava-se no segundo corte e bisada ( 24 meses em campo), assim realizou-se a colheita manual após despalha com fogo em maio de 2009.

$\mathrm{Na}$ alocação da malha experimental foram definidas as direções x e y do sistema de coordenadas cartesianas, sendo constituída de 120 pontos amostrais, distribuídos numa área de 14,53 $\mathrm{ha}^{-1}$ (418,46 m x 349,00 m). O espaçamento utilizado entre os pontos na grande malha foi de $42 \mathrm{~m} \mathrm{x} 43 \mathrm{~m}$, enquanto na malha de refinamento, construída com a finalidade de detectar alcances da dependência espacial para espaçamentos menores do que $42 \mathrm{~m}$, composta por três pontos adjacentes ao ponto central, o espaçamento foi de $9 \mathrm{~m}$ (Figura 1).

${ }^{4}$ Alves MC, Carvalho MP, Lima RC \& Vanzela SL (2009) Ambiente de produção para a cana-de-açúcar cultivada nas propriedades agrícolas da Usina Vale do Paraná S/A Álcool e Açúcar. Estudo Agrotécnico da Usina Vale do Paraná S/A Álcool e Açúcar - Suzanápolis (SP). Ilha Solteira. 107p. 
Foram pesquisados atributos do solo e da planta, individualmente coletados no entorno de cada ponto amostral da malha experimental. Os atributos do solo pesquisados foram a resistência à penetração (RP), umidade gravimétrica (UG) e o teor de matéria orgânica (MO), analisados nas camadas de 0-0,20 m (RP1, UG1 e MO1) e 0,20-0,40 m (RP2, UG2 e MO2). A RP foi avaliada com o penetrômetro de impactos (Modelo IAA/PlanalsucarStolf) uma única vez em cada ponto e calculada segundo a a expressão contida em Dalchiavon et al.(2011):

$\mathrm{RP}=\{5,581+6,891 .[\mathrm{N} /(\mathrm{P}-\mathrm{A}) \cdot 10]\} .0,0981$

Em que RP é a resistência do solo à penetração (MPa), $\mathbf{N}$ é o número de impactos efetuados com o martelo do penetrômetro para a obtenção da leitura no sabre e $\mathbf{A}$ e $\mathbf{P}$ são, respectivamente, as leituras de penetração no solo antes e depois da realização dos impactos $(\mathrm{cm})$.

Para a determinação da UG $\left(\mathrm{kg} \mathrm{kg}^{-1}\right)$ foram coletadas amostras deformadas de solo com trado de caneco com dimensões de $0,10 \mathrm{~m}$ de diâmetro por $0,20 \mathrm{~m}$ de altura (Embrapa, 1997). O teor de MO foi obtido através do carbono orgânico pelo método da combustão úmida, via colorimétrica, a partir da seguinte expressão (Raij et al., 2001):

$\mathrm{MO}=\mathrm{C} \times 1,724 \times 10$

Em que MO é o teor de matéria orgânica $\left(\mathrm{g} \mathrm{dm}^{-3}\right)$ e Cé o teor de carbono orgânico. As amostras de solo foram analisadas nos laboratórios de Física e de Fertilidade do Solo da Universidade Estadual Paulista, Ilha Solteira-SP.

A produtividade de colmos de cana-de-açúcar (PRO) foi obtida pela pesagem dos colmos contidos numa área útil de $9 \mathrm{~m}^{2}$ ( $3 \mathrm{~m} \times 3 \mathrm{~m}$ ), sendo duas linhas de $3 \mathrm{~m}$ por ponto amostral. Para cada atributo estudado efetuou-se a análise descritiva clássica, com auxílio do software estatístico SAS (Schlotzhaver \& Littell, 1997), em que foram calculados a média, a mediana, os valores mínimos e máximos, o desvio-padrão, o coeficiente de variação, a curtose, a assimetria e a distribuição de frequência. Posteriormente, realizou-se a identificação dos outliers, efetuando a substituição pelo valor médio dos circunvizinhos contidos na malha, conforme Dalchiavon et al. (2013). Para testar a hipótese da normalidade, ou da lognormalidade, realizouse o teste de Shapiro \& Wilk (1965) a 5\% de significância.

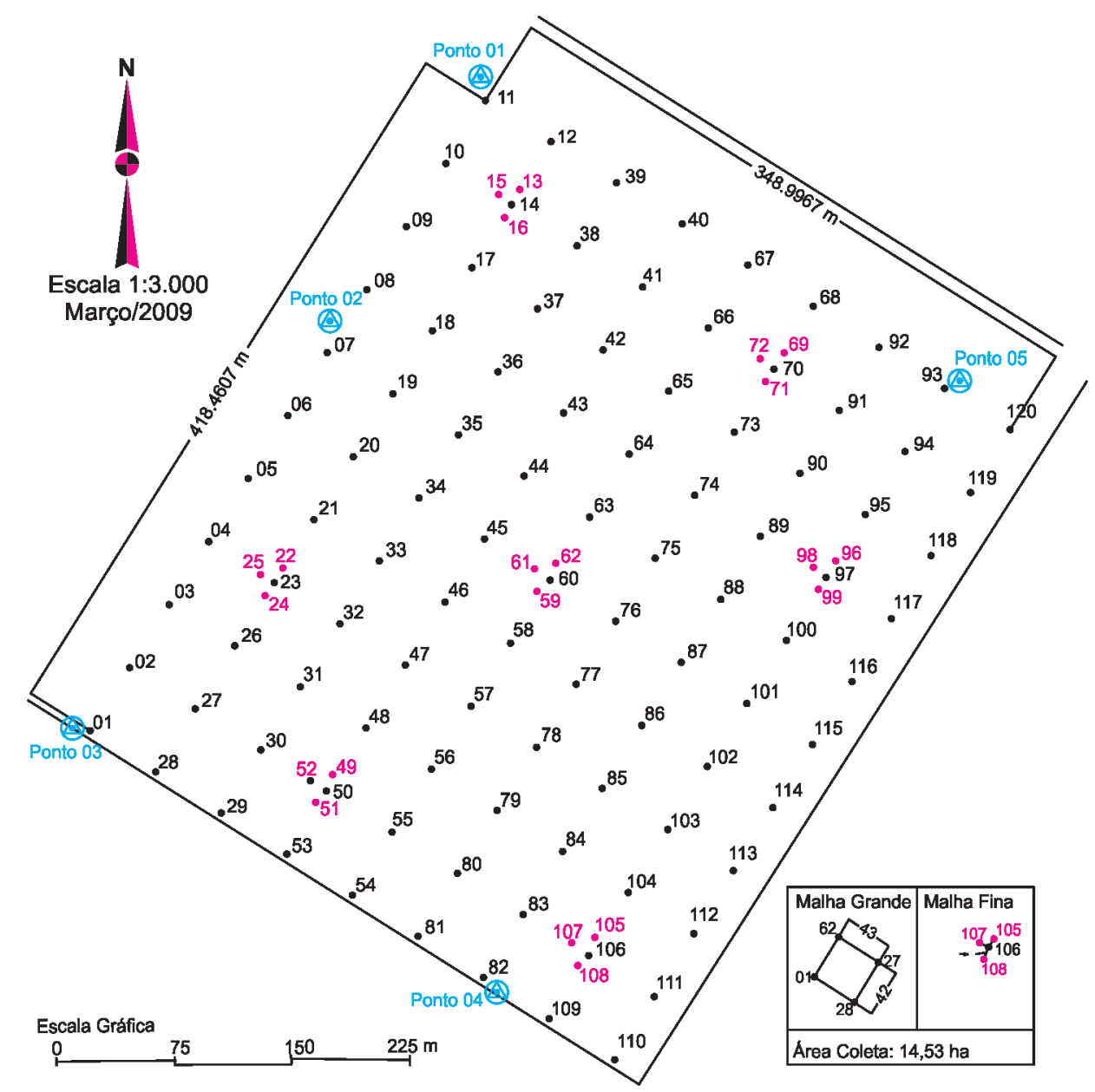

Figura 1. Croqui da malha experimental de amostragem. 
Foi montada a matriz de correlação, objetivando efetuar as correlações lineares simples para as combinações, duas a duas, entre todos os atributos estudados, a partir das quais foram efetuadas as modelagens das regressões lineares simples com a planilha de cálculos Excel. Por outro lado, utilizando-se o pacote computacional SAS ("stepwise"), a 5\% de probabilidade para a inclusão e exclusão de variáveis no modelo, foi efetuada a regressão linear múltipla da PRO (variável dependente) em razão dos demais atributos pesquisados (variáveis independentes) no intuito de selecionar aqueles que, nos devidos casos, proporcionariam a melhor equação para estimar a PRO.

Isoladamente para cada atributo, foi analisada sua dependência espacial pelo cálculo da semivariância. Contudo, para aqueles que apresentaram interdependência espacial, calcularam-se também seus semivariogramas cruzados, com base nos pressupostos de estacionaridade da hipótese intrínseca pelo uso do pacote Gamma Design Software 7.0 (GS $\left.{ }^{+}, 2004\right)$. Os ajustes dos semivariogramas simples e cruzados, em razão de seus modelos, foram efetuados prioritariamente pela seleção inicial de: 1) menor soma dos quadrados dos desvios (RSS), 2) maior coeficiente de determinação $\left(\mathrm{r}^{2}\right)$ e 3 ) maior avaliador da dependência espacial (ADE). Entretanto, para os atributos (ATR) que não apresentaram dependência espacial, isto é, na ausência de estacionaridade, retirou-se a tendência dos dados por meio da técnica da regressão múltipla polinomial (Dalchiavon et al., 2011). A decisão final do modelo que representou o ajuste foi tomada pela validação cruzada, assim como para a definição do tamanho da vizinhança que proporcionou a melhor malha de krigagem e/ou cokrigagem, realizada por meio da krigagem em blocos. Para cada atributo foram determinados o efeito pepita $\left(\mathrm{C}_{\mathrm{o}}\right)$, o alcance $\left(\mathrm{A}_{\mathrm{o}}\right)$ e o patamar $\left(\mathrm{C}_{\mathrm{o}}+\mathrm{C}\right)$. A análise do avaliador da dependência espacial (ADE) foi realizada mediante sugestões de Cambardella et al. (1994), modificada pelo $\mathrm{GS}^{+}$(2004), conforme a seguinte expressão:

$\mathrm{ADE}=\left[\mathrm{C} /\left(\mathrm{C}+\mathrm{C}_{\mathrm{o}}\right)\right] .100$

Em que ADE é o avaliador da dependência espacial; $\mathbf{C}$ é a variância estrutural; e $\mathbf{C}+\mathbf{C}_{\mathbf{0}}$ é o patamar. A interpretação proposta para o ADE foi conforme Dalchiavon \& Carvalho (2012): a) ADE < 20\% = variável espacial de muito baixa dependência (MB); b) $20 \% \leq \mathrm{ADE}<40 \%=$ baixa dependência (BA); c) 40\% $\leq \mathrm{ADE}<60 \%=$ média dependência (ME); d) $60 \% \leq \mathrm{ADE}<80 \%=$ alta dependência $(\mathrm{AL})$; e e) $80 \% \leq \mathrm{ADE}<100 \%=$ muito alta dependência (MA).

Para a validação cruzada obteve-se o número ideal de vizinhos (coeficiente $b$ mais próximo da unidade), e por meio da interpolação foram obtidos, os mapas de krigagem e de cokrigagem para análise da dependência e da interdependência espacial entre os atributos.

\section{RESULTADOS E DISCUSSÃO}

A umidade gravimétrica (UG1 e UG2) e a matéria orgânica (MO1) apresentaram média variabilidade nos dados, ao passo que a produtividade de colmos (PRO), resistência à penetração $(\mathrm{RP} 2)$ e MO2 tiveram variabilidade alta $\mathrm{e}$ muito alta para RP1 (Tabela 1). No geral, os dados apresentaram-se de maneira semelhante com aqueles de Corá et al. (2004), Souza et al. (2004a), Carvalho et al. (2008), Souza et al. (2008), Montanari et al. (2010,2012) e Dalchiavon et al. (2011). Cabe destacar a heterogenidade do solo em questão avaliada pelos altos coeficientes de variação, principalmente para as RPs, pois o solo ainda apresentava herança do preparo inicial, consequentemente interferindo na produtividade de colmos da área.

A PRO, RP1 e UG1 demonstraram distribuição de frequência normal com p-valor variando entre 0,108 e 0,664 (Tabela 1). Souza et al. (2004a) também observaram distribuição de frequência normal para a RP e UG, assim como Souza et al. (2008) para a PRO, também em cultivo de cana-de-açúcar. Segundo Cambardella et al. (1994), isso pode ser um indicativo de que as medidas de tendência central não refletem valores atípicos na distribuição.

A PRO obtida está de acordo com a média brasileira (Tabela 1), conforme dados da Unica (2009). Entretanto, era de se esperar uma PRO mais elevada, haja vista que se trata de cana com 24 meses. Por outro lado, foi 13,5\% superior à produtividade obtida por Souza et al. (2008), com a variedade SP80-1816, num Latossolo VermelhoAmarelo. A baixa PRO deve-se em grande parte ao Argissolo de apresentar textura arenosa no horizonte superficial e, consequentemente, baixa CTC e disponibilidade hídrica, o que resultou num baixo perfilhamento (média de 6 a 7 perfilhos $\mathrm{m}^{-1}$, quando o esperado para a variedade em questão seria de pelo menos 12 a 15 perfilhos $\mathrm{m}^{-1}$ ).

Verificou-se que a RP apresentou aumento de valores em profundidade (Tabela 1$)$ tidos como moderados $(1,0 \leq$ $\mathrm{RP}<2,0 \mathrm{MPa}$ ) pela classificação de Arshad et al. (1996). Uma vez que a microporosidade média desse solo é de $0,306 \mathrm{~m}^{3} \mathrm{~m}^{-3}$ e a densidade média de $1,565 \mathrm{~kg} \mathrm{dm}^{-3}$ (Alves et al., 2009) e no momento da avaliação da RP a UG média foi de $0,114 \mathrm{~kg} \mathrm{~kg}^{-1}$, isso resultou na umidade volumétrica de $0,178 \mathrm{~m}^{3} \mathrm{~m}^{-3}$. Esse valor $\left(0,178 \mathrm{~m}^{3} \mathrm{~m}^{-3}\right)$ representou $58,2 \% \mathrm{da}$ microporosidade $\left(0,306 \mathrm{~m}^{3} \mathrm{~m}^{-3}\right)$, confirmando que os dados de RP foram coletados num teor de água ideal, que é de 2/3 da microporosidade, e que, portanto, podem perfeitamente ser comparados aos da literatura.

Dessa forma, os dados observados para a RP diferiram daqueles de Souza et al. (2006), que constataram em 
seu estudo, para um Latossolo Vermelho distrófico, valores altos de $\mathrm{RP}(2,0 \leq \mathrm{RP}<4,0 \mathrm{MPa})$ para um conteúdo de água no solo em torno de $0,200 \mathrm{~kg} \mathrm{~kg}^{-1}$. Valores distintos também foram mencionados por Carvalho et al. (2008), em estudo desenvolvido num Latossolo Vermelho argiloso sob cultivo de cana-de-açúcar, cujos valores de RP verificados estavam acima de 4,0 MPa (muito alto) para um conteúdo de água no solo variando entre 0,240 e $0,260 \mathrm{~kg} \mathrm{~kg}^{-1}$, indicando, portanto, alta compactação do solo, o que poderá acarretar restrição ao desenvolvimento da cultura.

Observa-se ainda (Tabela 1) que a MO apresentou baixo teor em ambas as profundidades do solo, discordando da média obtida por Corá et al. (2004) para a camada de 0-0,20 m, contudo num Latossolo Vermelho. Em contrapartida, assemelham-se aos valores obtidos por Souza et al. (2010), que pesquisaram os atributos de um Latossolo Vermelho distrófico cultivado com cana-de-açúcar. A baixa amplitude nos valores de MO1 e MO2 justifica-se por se tratar de área cujo cultivo anterior era braquiária (extensiva não adubada), principalmente por ter sido realizado o revolvimento no preparo do solo com aivecas no ciclo anterior.
No estudo das correlações lineares de Pearson da PRO com os atributos do solo (Tabela 2), a PRO firmou correlações positivas e altamente significativas $(\mathrm{n}=120)$ com a RP1 ( $\left.r=0,185^{*}\right), \mathrm{UG} 2\left(\mathrm{r}=0,235^{* *}\right)$ e MO1 $\left(\mathrm{r}=0,236^{* *}\right)$, concordando com aquelas de Souza et al. (2008), com exceção da correlação entre a PRO e a RP1, que se apresentou positiva. Entretanto, as correlações PRO x RP1, PRO x UG2 e PRO x MO1 podem ser explicadas da seguinte maneira: a RP1 apresentou-se de forma moderada, que por sua vez, provavelmente pelo aumento, pode ter beneficiado o contato solo-raiz, por isso sua correlação positiva; a correlação foi positiva com a UG2 leva-se a inferir que houve beneficio de aumento na disponibilidade hídrica (solo arenoso) e, portanto, provável melhoria na absorção de nutrientes da solução do solo, uma vez que também o incremento da MO1 evidenciou liberação de nutrientes. As correlações RP1 x MO1 e RP2 x MO2 evidenciaram efeitos negativos e significativos, indicando que o aumento da MO do solo reduz a RP, também evidenciado pelos autores supracitados.

No âmbito das regressões lineares simples (Figuras 2 e 3), foram modeladas equações lineares (PRO x MO1; RP1 x MO1), potenciais (PRO x RP1; RP2 x MO2) e

Tabela 1. Análise descritiva da produtividade da cana-de-açúcar e de alguns atributos físico-químicos de um Argissolo Vermelho eutrófico da Fazenda Caiçara (Suzanápolis-SP)

\begin{tabular}{|c|c|c|c|c|c|c|c|c|c|c|}
\hline \multirow{3}{*}{ Atributo $^{(1)}$} & \multicolumn{10}{|c|}{ Medidas estatísticas descritivas } \\
\hline & \multirow[b]{2}{*}{ Média } & \multirow[b]{2}{*}{ Mediana } & \multicolumn{2}{|c|}{ Valor } & \multirow{2}{*}{$\begin{array}{l}\text { Desvio } \\
\text { Padrão }\end{array}$} & \multicolumn{3}{|c|}{ Coeficiente } & \multicolumn{2}{|c|}{$\begin{array}{c}\text { Probabilidade } \\
\text { do teste }^{(2)}\end{array}$} \\
\hline & & & Mínimo & Máximo & & $\begin{array}{c}\text { Variação } \\
(\%)\end{array}$ & Curtose & Assimetria & $\operatorname{Pr}<w$ & DF \\
\hline PRO (t ha $\left.{ }^{-1}\right)$ & 89,2 & 90,0 & 11,2 & 142,2 & 24,4 & 27,3 & 0,677 & $-0,401$ & 0,108 & NO \\
\hline RPl (MPa) & 1,066 & 1,092 & 0,349 & 1,907 & 0,324 & 30,4 & $-0,335$ & 0,132 & 0,664 & NO \\
\hline $\mathrm{RP2}(\mathrm{MPa})$ & 1,629 & 1,543 & 0,746 & 2,857 & 0,428 & 26,3 & $-0,049$ & $-0,030$ & 0,109 & LN \\
\hline UGl $\left(\mathrm{kg} \mathrm{kg}^{-1}\right)$ & 0,114 & 0,115 & 0,074 & 0,143 & 0,014 & 12,2 & $-0,031$ & $-0,252$ & 0,652 & No \\
\hline UG2 ( $\left.\mathrm{kg} \mathrm{kg}^{-1}\right)$ & 0,114 & 0,113 & 0,086 & 0,147 & 0,013 & 11,1 & 0,268 & 0,437 & 0,044 & TN \\
\hline $\operatorname{MOl}\left(\mathrm{g} \mathrm{dm}^{-3}\right)$ & 15,5 & 15,0 & 10,0 & 23,0 & 2,87 & 18,5 & 0,003 & 0,212 & 0,012 & TN \\
\hline $\mathrm{MO2}\left(\mathrm{g} \mathrm{dm}^{-3}\right)$ & 14,4 & 14,0 & 7,0 & 23,0 & 3,00 & 20,9 & 0,126 & $-0,241$ & 0,013 & $\mathrm{TL}$ \\
\hline
\end{tabular}

(1) PRO, RP, UG e MO são, respectivamente, a produtividade de colmos por hectare, resistência à penetração, umidade gravimétrica e o teor de matéria orgânica. Os índices 1 e 2 são, respectivamente, as profundidades de $0-0,20$ e $0,20-0,40$ m. ${ }^{(2)} \mathrm{DF}=$ distribuição de frequência (Shapiro-Wilk), sendo NO, LN, TN e TL, respectivamente, do tipo normal, lognormal, tendendo a normal e tendendo a lognormal.

Tabela 2. Matriz de correlação da produtividade da cana-de-açúcar e de alguns atributos físico-químicos de um Argissolo Vermelho eutrófico da Fazenda Caiçara (Suzanápolis-SP)

\begin{tabular}{|c|c|c|c|c|c|c|}
\hline \multirow{2}{*}{ Atributos $^{(1)}$} & \multicolumn{6}{|c|}{ Coeficiente de correlaçã $0^{(2)}$} \\
\hline & PRO & RPl & RP2 & UGl & UG2 & MOl \\
\hline RPl & $0,185^{*}$ & & & & & \\
\hline RP2 & 0,044 & $0,336^{* *}$ & & & & \\
\hline UGl & 0,171 & $-0,054$ & $-0,098$ & & & \\
\hline UG2 & $0,235^{* *}$ & $-0,094$ & 0,013 & $0,287^{* *}$ & & \\
\hline MO1 & $0,236^{* *}$ & $-0,264^{* *}$ & $-0,082$ & 0,123 & 0,172 & \\
\hline MO2 & 0 & $-0,260^{* *}$ & $-0,267 * *$ & 0,074 & 0,143 & $0,440^{* *}$ \\
\hline
\end{tabular}

(1) Vide Tabela 1. ${ }^{(2)} * *$ significativo a $1 \%$ e $*$ significativo a $5 \%$ de probabilidade. 
exponencial (PRO x UG2). Foi constatada relação positiva entre a PRO x RP1 e PRO x UG2, indicando que um aumento na RP e na UG refletirá positivamente na PRO (Figura 2a,b), pois aumento na RP tem reflexo direto na microporosidade do solo e, portanto, melhoria na disponibilidade hídrica, permitindo avanço na absorção de nutrientes da solução do solo. Dessa forma, verifica-se que para a condição de máxima compactação do solo observada no presente estudo (RP1 = 1,907 MPa; Tabela 1), a PRO estimada a partir da equação $y=87,313 . x^{(0,173 *)}(r=0,212 *)$ foi de $97,6 \mathrm{t} \mathrm{ha}^{-1}$. Analogamente, considerando o teor de máxima UG2 do solo igual a $0,147 \mathrm{~kg} \mathrm{~kg}^{-1}$, a equação y $=48$,993.e ${ }_{(5,07 * * \cdot x)}\left(\mathrm{r}=0,237^{* *}\right)$ estimou uma PRO de 103,3 $\mathrm{tha}^{-1}$.

A regressão da PRO x MO1 $\left(r=0,207^{*}\right)$ indicou que a variação dos teores da MO1 entre 10 e $23 \mathrm{~g} \mathrm{dm}^{-3}$ implicará numa variação de PRO compreendida entre 80,1 e 104,3 t
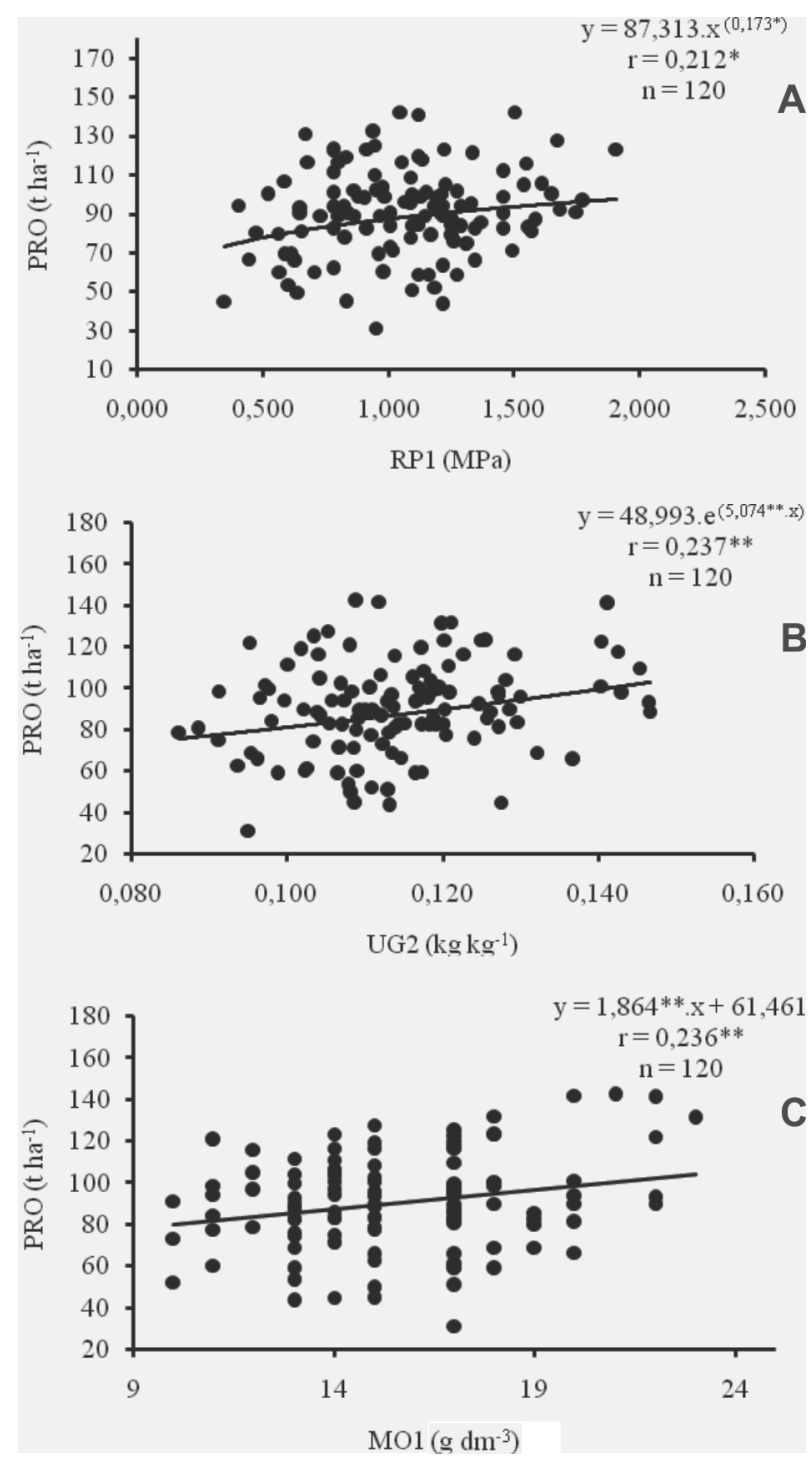

Figura 2. Equações de regressão da produtividade da cana-deaçúcar em razão de alguns atributos físico-químicos de um Argissolo Vermelho eutrófico da Fazenda Caiçara (Suzanápolis-SP). ha $^{-1}$ (Figura 2c), ao passo que para o teor médio da MO1 $\left(15,5 \mathrm{~g} \mathrm{dm}^{-3}\right)$ ocorrerá uma PRO estimada em 90,4 t ha-1. Contudo, tal equação destina-se às estimativas da produtividade apenas para essa amplitude de dados, aproximando-se muito da equação de Vitti et al. (2008), que também encontraram relação linear positiva entre tais variáveis, sendo $\mathrm{y}=50,9 \cdot \mathrm{x}+23,4\left(\mathrm{r}=0,934^{* *}\right)$, com valores da MO expressos em porcentagem. Assim, considerando o teor médio para a variável independente (MO) obtido neste estudo pela equação dos referidos autores, seria possível estimar uma PRO média de 102,3 t ha-1 ${ }^{-1}$ ou seja, 11,9 t ha-1 a mais que a primeira equação. Tais equações possuem relevância no âmbito do manejo e da conservação do solo, haja vista que a MO influencia substancialmente suas propriedades químicas, físicas e biológicas.

Em relação às regressões de comportamento inverso, o aumento linear nos teores de MO melhorou substancialmente a condição física do solo, pois diminuiu a sua compactação, quando avaliada pela RP, sendo o inverso perfeitamente verdadeiro (Figuras 3a,b). Tais observações estão de acordo com aquelas citadas por Melo et al. (2008) para um Neossolo Litólico com vegetação de caatinga. Assim, no presente estudo, quando a MO1 variou entre 10,0 e 23,0 $\mathrm{g} \mathrm{dm}^{-3}$, a RP1 oscilou entre 1,237 e 0,847 MPa (Figura 3a), da mesma forma em que a RP2 variou potenci-
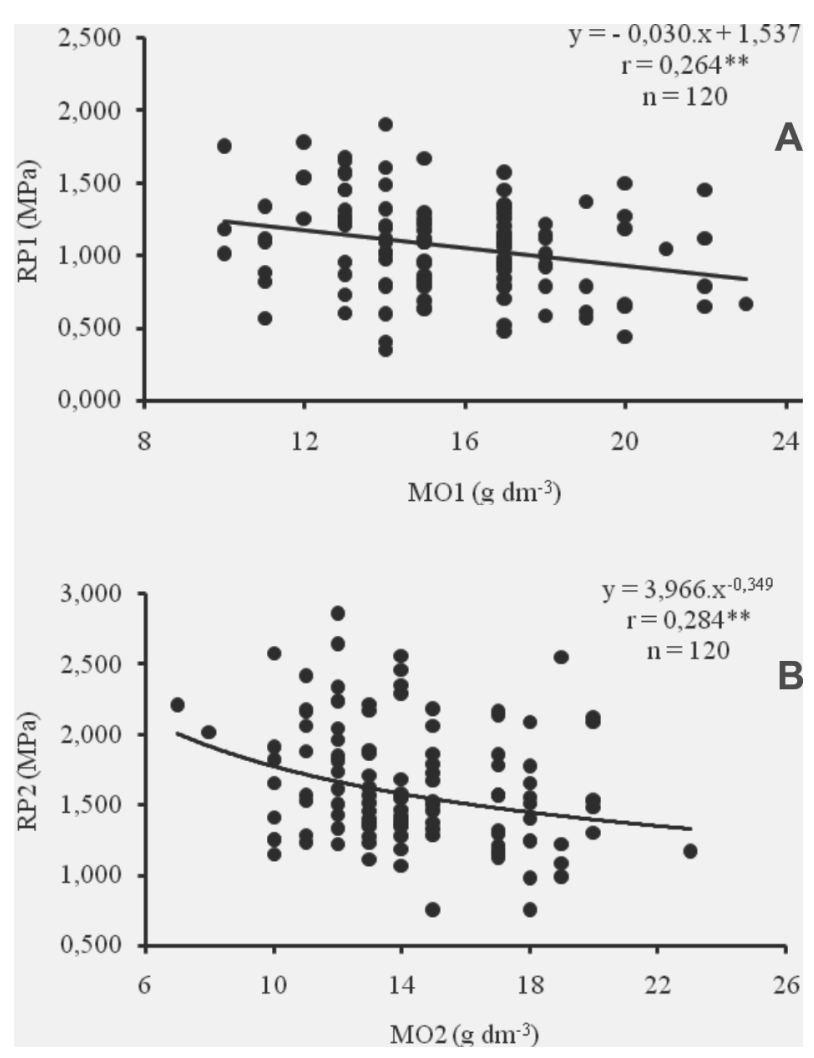

Figura 3. Equações de regressão da resistência à penetração em razão dos teores de matéria orgânica de um Argissolo Vermelho eutrófico da Fazenda Caiçara (Suzanápolis-SP). 
Inter-relações da produtividade de cana soca com a resistência à penetração, umidade e matéria...261

almente entre 2,011 e 1,328 MPa quando a MO2 oscilou entre 7,0 e 23,0 $\mathrm{g} \mathrm{dm}^{-3}$ (Figura 3b).

$\mathrm{Na}$ análise da regressão linear múltipla da PRO em função dos demais atributos, o modelo testado (Equação 4) explicou aproximadamente $16,6 \%$ da variação da produtividade de colmos de cana-de-açúcar $\left(\mathrm{R}^{2}=0,166^{* *}\right)$. Os coeficientes das variáveis independentes RP1 e MO1 foram significativos a $1 \%(* *)$ e a UG2 a $5 \%$ (*) de probabilidade.

PRO $\left(\mathrm{t} \mathrm{ha}^{-1}\right)=-6,29+19,07 \cdot \mathrm{RP} 1 * *+2,15 \cdot \mathrm{MO} 1^{* *}+$ $+376,04 . \mathrm{UG} 2 *$

Com exceção da UG2 e da MO2, que apresentaram efeito pepita puro, talvez por decorrência do preparo inicial do solo, todos os demais atributos tiveram dependência espacial, evidenciando que suas distribuições no espaço não foram aleatórias (Tabela 3). Verifica-se, nos semivariogramas simples, que o coeficiente de determinação espacial $\left(\mathrm{r}^{2}\right)$ decresceu na seguinte ordem: (1) RP1, (2) RP2, (3) UG1, (4) PRO e (5) \#MO1. Assim, em relação aos dois primeiros, que tiveram os mais elevados $r^{2}$, observou-se o seguinte: RP1, seu $\mathrm{r}^{2}$ indicou ser o modelo de melhor ajuste, apresentando ADE muito alto, com alcance de 144,0 metros e modelo exponencial discordando de Souza et al. (2010), que ajustaram semivariograma esférico, com coeficiente de determinação espacial de 0,870 , ADE de $51,0 \%$ e alcance de $84 \mathrm{~m}$. Por outro lado, o ${ }^{2}$ do RP2 indicou ser este o segundo atributo de melhor ajuste semivariográfico, cujo ADE observado foi médio, com o alcance de 60,0 metros e ajuste gaussiano. Portanto, a resistência à penetração mostrou-se como potencial indicador da qualidade física do solo.

A relação decrescente dos alcances para os semivariogramas simples foi: (1) \#MO1, (2) RP1, (3) UG1, (4) PRO e (5) RP2, demonstrando que para manejos específicos e localizados os valores dos alcances a serem utilizados como referência em trabalhos futuros não deverão ser menores do que $60 \mathrm{~m}$, por representarem a distância dentro da qual os valores de determinado atributo são iguais entre si (Tabela 3).

Os mapas de krigagem (PRO, UG1 e £MO1) apresentaram elevada correlação espacial direta (Figura 4). Dessa forma, de maneira geral, nos dois terços horizontais inferiores e na região nordeste (Figura $4 b$ ), ocorreram os mais elevados valores da PRO $\left(89,8 \mathrm{a}>115,6 \mathrm{t} \mathrm{ha}^{-1}\right)$. Por outro lado, no primeiro terço (vertical e horizontal) e na região leste foi verificado predominância de valores variando entre $0,115 \mathrm{e}>0,126 \mathrm{~kg} \mathrm{~kg}^{-1}$ para a UG1 (Figura 4d). AMO1 em praticamente todos os sítios do mapa mostrou predominância dos valores mais elevados (16,4 a > 19,0 $\left.\mathrm{g} \mathrm{dm}^{-3}\right)$ (Figura 4f). Em contrapartida, em relação aos referidos mapas de krigagem ocorreu o inverso nas demais regiões, isto é, os mais baixos valores da PRO (55,8-89,9 $\left.\mathrm{t} \mathrm{ha}^{-1}\right)$, UG1 (0,100-0,115 kg kg-1) e £MO1 (12,8-16,4 $\left.\mathrm{g} \mathrm{dm}^{-3}\right)$.

A cokrigagem entre a PRO e UG1, com modelo gaussiano, teve alcance de $110,0 \mathrm{~m}$ e ADE muito alto, evidenciando que $61,6 \%$ da variabilidade espacial da PRO foi explicada pela variabilidade espacial da UG1 (Tabela 3, Figura 5a,b). Assim, pôde-se constatar que onde ocorreram os maiores valores da UG1 (Figura 4d) foram mapeados os maiores valores da PRO (Figuras $4 \mathrm{~b}$ e 5b), sendo o inverso verdadeiro. Por conseguinte, do ponto de vista espacial da área pesquisada, nos sítios em que a UG1 variar entre $0,115-0,126 \mathrm{~kg} \mathrm{~kg}^{-1}$, a PRO esperada estará compreendida entre 88,0-128,0 t ha-1. Por outro lado, naqueles em que a UG1 estiver entre $0,100-0,115 \mathrm{~kg} \mathrm{~kg}^{-1}$ a PRO esperada estará compreendida entre 35,0-88,0 t ha-1, confirmando a grande influência da UG sobre a produtividade de cana em solo arenoso, principalmente numa região onde os déficits hídricos são comuns, como é o caso da região noroeste do Estado de São Paulo.

Tabela 3. Parâmetros dos semivariogramas simples e cruzados da produtividade da cana-de-açúcar e de alguns atributos físicoquímicos de um Argissolo Vermelho eutrófico da Fazenda Caiçara (Suzanápolis-SP)

\begin{tabular}{|c|c|c|c|c|c|c|c|c|c|c|c|}
\hline \multirow{3}{*}{ Atributo $^{(1)}$} & \multicolumn{11}{|c|}{ Parâmetros do ajuste } \\
\hline & \multirow{2}{*}{ Modelo $^{(2)}$} & \multirow{2}{*}{$\mathrm{C}_{0}$} & \multirow{2}{*}{$\mathrm{C}_{0}+\mathrm{C}$} & \multirow{2}{*}{$\begin{array}{l}\mathbf{A}_{0} \\
(\mathrm{~m})\end{array}$} & \multirow{2}{*}{$\mathbf{r}^{2}$} & \multirow{2}{*}{$\mathrm{SQR}^{(3)}$} & \multicolumn{2}{|c|}{$\mathbf{A D E}^{(4)}$} & \multicolumn{3}{|c|}{ Validação cruzada } \\
\hline & & & & & & & $\%$ & Classe & $\mathbf{a}$ & b & $\mathbf{r}$ \\
\hline \multicolumn{12}{|c|}{$\gamma(h)$ simples } \\
\hline PRO & $\operatorname{gau}(57)$ & $1,97 \cdot 10^{-2}$ & $4,86.10^{-2}$ & 72,0 & 0,854 & $7,82.10^{3}$ & 59,4 & ME & $6,5.10$ & 0,281 & 0,122 \\
\hline RP1 & $\exp (72)$ & $1,35.10^{-2}$ & $6,16 \cdot 10^{-2}$ & 144,0 & 0,947 & $1,24.10^{-4}$ & 85,3 & MA & $4,8.10^{-1}$ & 0,555 & 0,318 \\
\hline RP2 & gau (50) & $7,80.10^{-2}$ & $1,72 \cdot 10^{-1}$ & 60,0 & 0,898 & $5,48 \cdot 10^{-4}$ & 54,7 & ME & 1,25 & 0,232 & 0,084 \\
\hline UG1 & $\exp (64)$ & $1,03.10^{-4}$ & $2,07.10^{-4}$ & 135,6 & 0,872 & $6,13 \cdot 10^{-40}$ & 50,2 & ME & $5.0 .10^{-2}$ & 0,5566 & 0,192 \\
\hline UG2 & epp & $1,67 \cdot 10^{-4}$ & $1,67 \cdot 10^{-4}$ & - & - & - & - & - & - & - & - \\
\hline \#MO1 & $\exp (236)$ & 3,89 & 7,78 & 182,7 & 0,709 & 1,57 & 50,0 & ME & $-5,0.10^{-2}$ & 0,785 & 0,270 \\
\hline MO2 & epp & 9,06 & 9,06 & - & - & - & - & - & - & - & - \\
\hline \multicolumn{12}{|c|}{$\gamma($ h) cruzado [planta $=f($ planta;solo $)]$} \\
\hline PRO=f(UGl) & gau (144) & $1,00.10^{-3}$ & $3,99 \cdot 10^{-2}$ & 110,0 & 0,616 & $9,41 \cdot 10^{-4}$ & 97,5 & MA & $7,1.10$ & 0,218 & 0,141 \\
\hline PRO=f(\#MO1) & $\exp (83)$ & $1,00.10^{-1}$ & $1,33.10$ & 165,9 & 0,653 & $4,18.10$ & 99,3 & MA & $7,4.10$ & 0,173 & 0,110 \\
\hline
\end{tabular}

(1) Vide Tabela 1. \# trabalhado com o resíduo do atributo. Parênteses sucedendo o modelo significa o número de pares no primeiro lag. (2) gau = gaussiano, exp = exponencial, epp = efeito pepita puro. ${ }^{(3)} \mathrm{SQR}=$ soma dos quadrados dos resíduos. ${ }^{(4)} \mathrm{ADE}=$ avaliador da dependência espacial, sendo $\mathrm{ME}=$ média e MA = muito alta. 


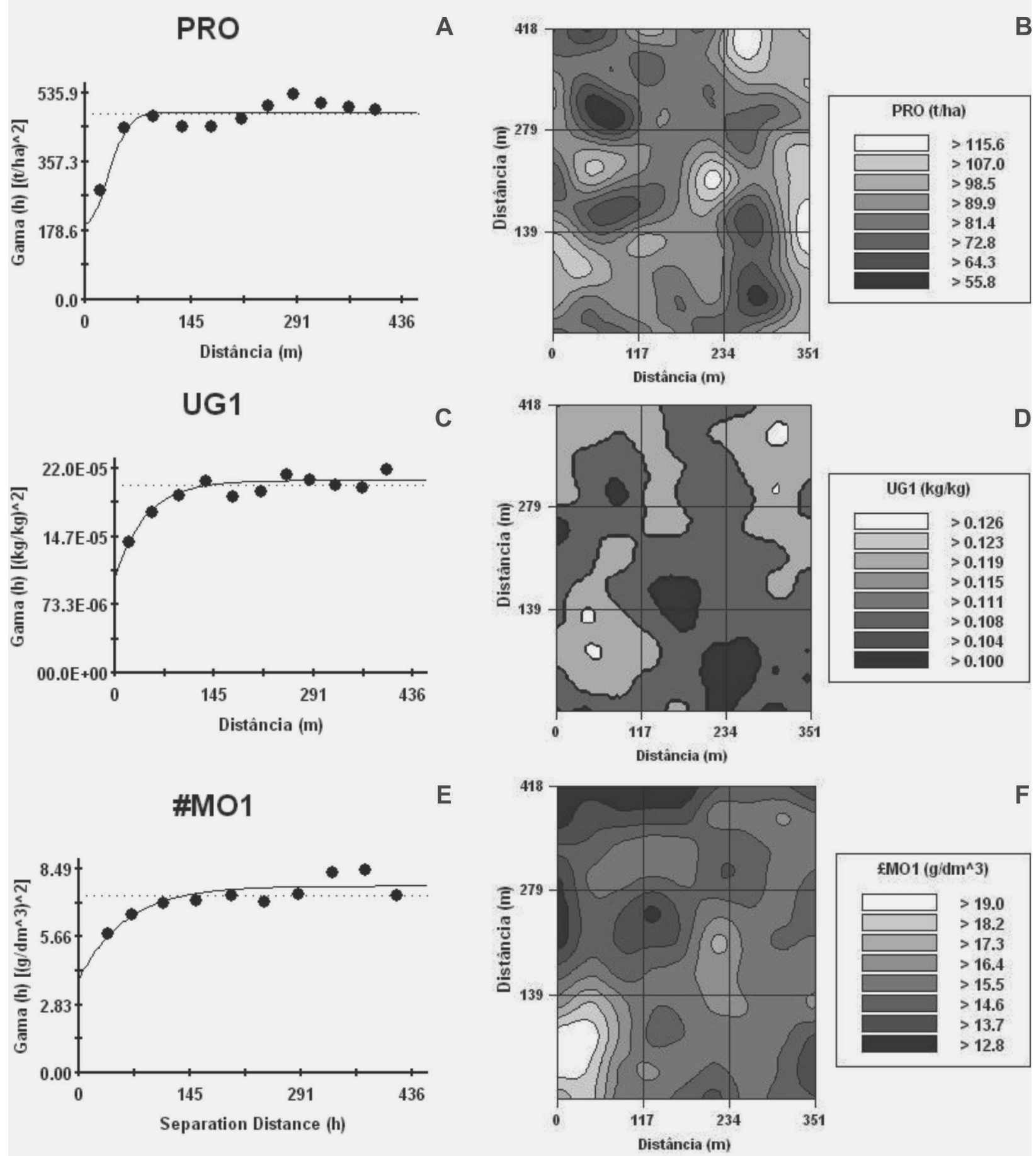

Figura 4. Semivariogramas simples e mapas de krigagem da produtividade da cana-de-açúcar da UG1 e da MO1 de um Argissolo Vermelho eutrófico da Fazenda Caiçara (Suzanápolis-SP).

Além disso, o semivariograma cruzado (modelo exponencial) e o mapa de cokrigagem da PRO em função da \#MO1 atestaram que a variabilidade espacial da \#MO1 explicou 65,3\% da variabilidade espacial da PRO (Tabela 3 ; Figura $5 \mathrm{c}, \mathrm{d}$ ), de maneira que nos sítios em que ocorreram os mais elevados valores da \#MO1 (16,4-19,0 $\left.\mathrm{g} \mathrm{dm}^{-3}\right)$ foram justamente aqueles em que a PRO apresentou os mais elevados valores $\left(88,0-128,0 \mathrm{t} \mathrm{ha}^{-1}\right)$, ao passo que onde ocorreram os mais baixos valores da \#MO1 $(12,8$ -
$15,5 \mathrm{~g} \mathrm{dm}^{-3}$ ) foram os locais em que a PRO apresentou os menores valores $\left(35,0-75,0 \mathrm{tha}^{-1}\right)$. Assim, foi observada alta dependência espacial da PRO com a \#MO1, sugerindo a importância de práticas agrícolas que visem à elevação de seus teores no solo, uma vez que ficaram claros seus benefícios à produtividade da cultura da cana-deaçúcar tanto na melhoria física (agregação e disponibilidade hídrica) quanto na fertilidade do solo (Souza et al., 2004b). 


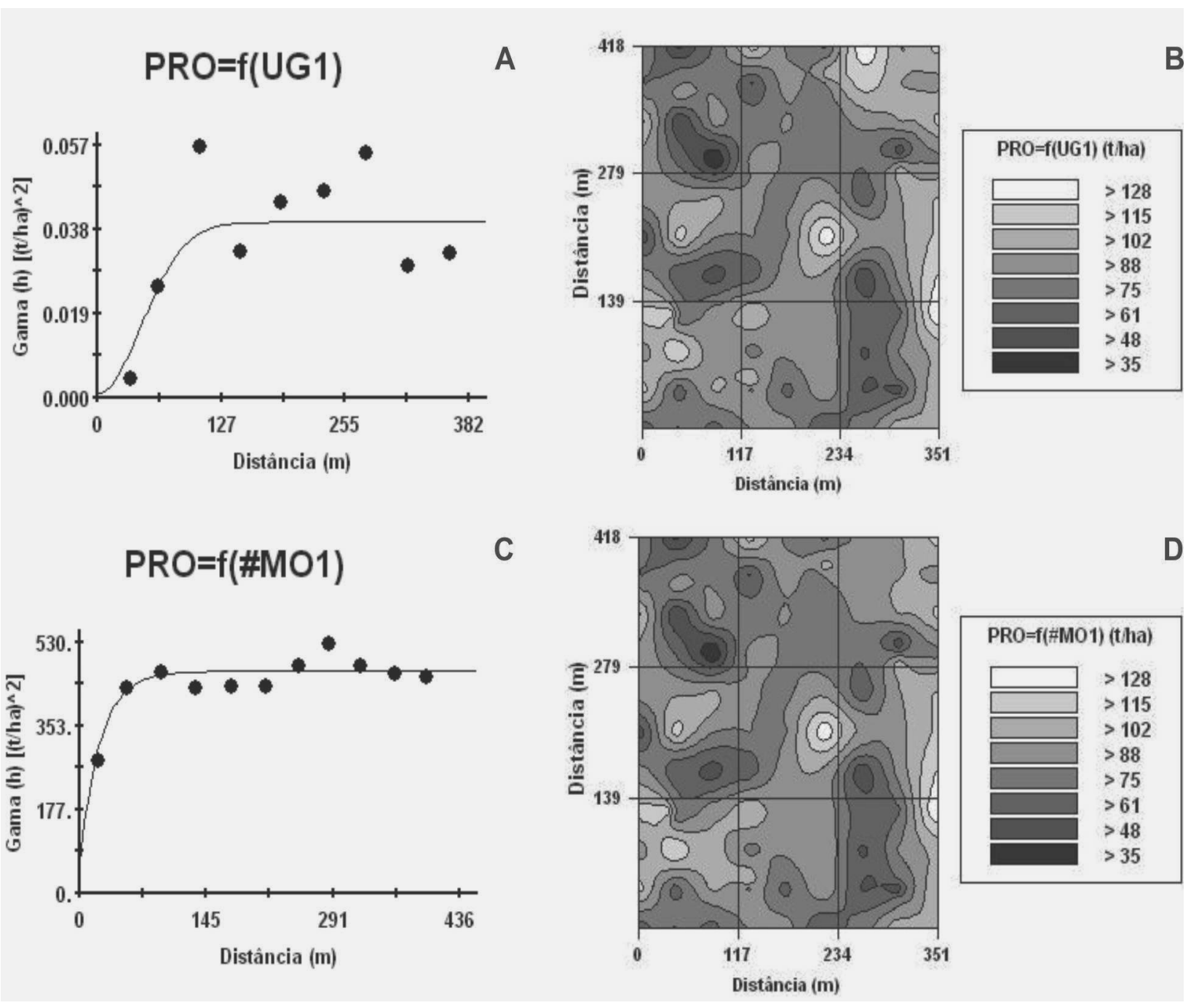

Figura 5. Semivariogramas cruzados e mapas de cokrigagem da PRO em razão da UG1 e da MO1 num Argissolo Vermelho eutrófico da Fazenda Caiçara (Suzanápolis-SP).

Não obstante, do ponto de vista espacial e do manejo do solo, pôde-se constatar que tanto o teor de água quanto o de matéria orgânica do solo apresentaram-se como bons indicadores da produtividade de colmos de canade-açúcar.

\section{CONCLUSÕES}

A matéria orgânica correlaciona-se linear e negativamente com a resistência à penetração, indicando que manejos do solo que visem seu aumento no perfil melhoram suas as condições físicas e, por conseguinte, o desenvolvimento e a produtividade da cana-de-açúcar.

A umidade gravimétrica e o teor de matéria orgânica do solo correlacionam-se de forma direta, linear (UG2 e MO1) e espacialmente (UG1 e MO1) com a produtividade da cana-de-açúcar, demonstrando serem os melhores atributos, dentre os pesquisados, para estimar e aumentar a sua produtividade agrícola.

\section{REFERÊNCIAS}

Arshad MA, Lower B \& Grossman B (1996) Physical tests for monitoring soil quality. In: Doran JW \& Jones AJ (Eds.) Methods for assessing soil quality. Soil Science Society of America Journal, 49:123-141. (Special Publication, 49).

Beutler NA \& Centurion JF (2003) Efeito do conteúdo de água e da compactação do solo na produção de soja. Pesquisa Agropecuária Brasileira, 38:849-856.

Cambardella CA, Moorman TB \& Novak JM (1994) Field-scale variability of soil properties in central Iowa soils. Science Society of America Journal, 58:1501-1511.

Carvalho LA, Neto VJM, Silva LF, Pereira JG, Nunes WAGA \& Chaves CHC (2008) Resistência mecânica do solo à penetração (RMP) sob cultivo de cana-de-açúcar, no município de Rio Brilhante-MS. Agrarian, 1:07-22.

Conab - Companhia Nacional de Abastecimento (2013) Indicadores da Agropecuária. Brasília, Conab. 19p.

Corá JE, Araujo AV, Pereira GT \& Beraldo JMG (2004) Variabilidade espacial de atributos do solo para adoção do sistema de agricultura de precisão na cultura de cana-de-açúcar. Revista Brasileira de Ciência do Solo, 28:1013-1021.

Rev. Ceres, Viçosa, v. 61, n.2, p. 255-264, mar/abr, 2014 
Dalchiavon FC \& Carvalho MP (2012) Correlação linear e espacial dos componentes de produção e produtividade da soja. Semina - Ciências Agrárias, 33:541-552.

Dalchiavon FC, Carvalho MP, Nogueira DC, Romano D, Abrantes FL, Assis JT \& Oliveira MS (2011) Produtividade da soja e resistência mecânica à penetração do solo sob sistema plantio direto no cerrado brasileiro. Pesquisa Agropecuária Tropical, 41:08-19.

Dalchiavon FC, Carvalho MP, Montanari R \& Andreotti M (2013) Strategy of specification of management areas: rice grain yield as related to soil fertility. Revista Brasileira de Ciência do Solo, $37: 45-54$.

Embrapa - Empresa Brasileira de Pesquisa Agropecuária (1997) Centro Nacional de Pesquisa de Solos. Manual de métodos de análise do solo. $2^{\mathrm{a}}$ ed. Rio de Janeiro, 212p.

$\mathrm{GS}^{+}$(2004) Geostatistics for environmental sciences. $7^{\mathrm{a}}$ ed Michigan, Gamma Design Software. 159p.

Lima CGR, Carvalho MP, Narimatsu KCP, Silva MG \& Queiroz HÁ (2010) Atributos físico-químicos de um Latossolo do cerrado brasileiro e sua relação com características dendrométricas do eucalipto. Revista Brasileira de Ciência do Solo, 34:163173.

Martins MV, Carvalho MP, Andreotti M \& Montanari R (2009) Correlação linear e espacial entre a produtividade do feijoeiro e atributos físicos de um LATOSSOLO VERMELHO Distroférrico de Selvíria, Estado de Mato Grosso do Sul. Acta Scientiarum Agronomy, 31:147-154.

Melo RO, Pacheco EP, Menezes JC \& Cantalice JRB (2008) Susceptibilidade à compactação e correlação entre as propriedades físicas de um Neossolo sob vegetação de caatinga. Caatinga, 21:12-17.

Montanari R, Carvalho MP, Andreotti M, Dalchiavon FC, Lovera LH \& Honorato MAO (2010) Aspectos da produtividade do feijão correlacionados com atributos físicos do solo sob elevado nível tecnológico de manejo. Revista Brasileira de Ciência do Solo, 34:1811-1822.

Montanari R, Marques Júnior J, Pereira GT \& Souza ZM (2005) Forma da paisagem como critério para otimização amostral de latossolos sob cultivo de cana-de-açúcar. Pesquisa Agropecuária Brasileira, 40:69-77.

Montanari R, Zambianco EC, Corrêa AR, Pellin DMP, Carvalho MP \& Dalchiavon FC (2012) Atributos físicos de um Latossolo Vermelho correlacionados linear e espacialmente com a consorciação de guandu com milheto. Revista Ceres, 59:125135 .
Raij BV, Andrade JC, Cantarella H \& Quaggio JÁ (2001) Análise química para avaliação da fertilidade de solos tropicais. Campinas, Instituto Agronômico. 285p.

Santos ML, Carvalho MP, Rapassi RMA, Muraishi CT, Maller A \& Matos FA (2006) Correlação linear e espacial entre produtividade de milho (Zea mays L.) e atributos físicos de um Latossolo Vermelho distroférrico sob plantio direto do Cerrado Brasileiro. Acta Scientiarum Agronomy, 28:313-321.

Schlotzhaver SD \& Littell RC (1997) SAS system for elementary statical analysis. $2^{\mathrm{a}}$ ed. Cary, SAS. 441p.

Shapiro SS \& Wilk MB (1965) An analysis of variance test for normality: Complete samples. Biometrika, 52:591-611.

Souza ZM, Campos MCC, Cavalcante ÍHL, Marques Júnior J, Cesarin LG \& Souza SR (2006) Dependência espacial da resistência do solo à penetração e do teor de água do solo sob cultivo contínuo de cana-de-açúcar. Ciência Rural, 36:128-134.

Souza ZM, Cerri PDG, Magalhães PG \& Campos MCC (2008) Correlação dos atributos físicos e químicos do solo com a produtividade de cana-de-açúcar. Revista de Biologia e Ciências da Terra, 8:183-190.

Souza ZM, Marques Júnior J \& Pereira GT (2010) Geoestatística e atributos do solo em áreas cultivadas com cana-de-açúcar. Ciência Rural, 40:48-56.

Souza ZM, Marques Júnior J \& Pereira GT (2004b) Variabilidade espacial da estabilidade de agregados e matéria orgânica em solos de relevos diferentes. Pesquisa Agropecuária Brasileira, 39:491-499.

Souza ZM, Marques Júnior J \& Pereira GT (2004a) Variabilidade espacial de atributos físicos do solo em diferentes formas do relevo sob cultivo de cana-de-açúcar. Revista Brasileira de Ciência do Solo, 28:937-944.

Trangmar BB, Yost RS, Wade MK \& Uehara G (1985) Applications of geostatistics to spatial studies of soil properties. Advances in Agronomy, 38:45-94.

Unica - União da Indústria de Cana-de-Açúcar (2009) Produção de cana-de-açúcar no Brasil. Disponível em: <http:// www.unica.com.br/dadosCotacao/estatistica/>. Acessado em: 20 de Setembro de 2009.

Utset A \& Cid G (2001) Soil penetrometer resistance spatial variability in a Ferralsol at several soil moisture conditions. Soil and Tillage Research, 61:193-202.

Vitti AC, Cantarella H, Trivelin PCO \& Rossetto R (2008) Nitrogênio. In: Dinardo-Miranda LL, Vasconcelos ACM \& Landell MGA (Eds.) Cana-de-açúcar. Campinas, FUNAG. p.239-270. 